\title{
A functional micro-needle sensor for voltammetric determination of iron in coastal waters
}

\author{
Haitao Han ${ }^{\mathrm{a}, \mathrm{b}}$, Dawei Pan ${ }^{\mathrm{a}, \mathrm{b}, \mathrm{c}, *}$, Fei Pan ${ }^{\mathrm{a}, \mathrm{c}}$, Xueping Hu ${ }^{\mathrm{d}}$, Rilong Zhu ${ }^{\mathrm{e}, *}$ \\ ${ }^{a}$ CAS Key Laboratory of Coastal Environmental Processes and Ecological Remediation, Shandong Key Laboratory of Coastal Environmental Processes, Research Center \\ for Coastal Environment Engineering Technology of Shandong Province, Yantai Institute of Coastal Zone Research, Chinese Academy of Sciences, Yantai 264003, PR \\ China \\ ${ }^{\mathrm{b}}$ Center for Ocean Mega-Science, Chinese Academy of Sciences, Qingdao 266071, PR China \\ ${ }^{\mathrm{c}}$ University of Chinese Academy of Sciences, Beijing 100049, PR China \\ ${ }^{\mathrm{d}}$ College of Chemistry and Chemical Engineering, Linyi University, Linyi 276000, PR China \\ ${ }^{\mathrm{e}}$ College of Chemistry and Chemical Engineering, Hunan University, Changsha 410082, PR China
}

\section{A R T I C L E I N F O}

\section{Keywords:}

Micro-needle sensor

Gold nanoclusters

Conducting polymer film

Iron

Coastal waters

\begin{abstract}
A B S T R A C T
A novel micro-needle sensor based on gold nanoclusters (AuNCs) immobilized with conducting polymer film poly (3,4-ethylenedioxythiophene)-poly(sodium 4-styrenesulfonate) (PEDOT-PSS) was fabricated for determination of iron in coastal waters. The unique aciform structure of the micro-needle electrode (MNE) provided larger specific surface area than common electrodes. The surface modification of MNE with PEDOT-PSS improved the conductivity of the electrode and increased the active sites for combination and immobilization of AuNCs. The cluster-like structure of AuNCs was formed as a result of the regulation of PEDOT-PSS, which showed excellent electrocatalytic activity for the reduction of $\mathrm{Fe}^{3+}$. The so-fabricated AuNCs/PEDOT-PSS/MNE showed excellent sensitivity, selectivity, reproducibility, and repeatability for iron determination with the linear range of $0.01-5 \mu \mathrm{M}$ and detection limit of $3.1 \mathrm{nM}$, respectively. Furthermore, the micro-needle sensor was successfully used for the determination of acidified dissolved iron in a coastal river from the source to the estuary with satisfactory results.
\end{abstract}

\section{Introduction}

As one of the common and essential metal micronutrients, iron (Fe) plays an important role in plant metabolism by participating in the electron transport, chlorophyll synthesis, nitrate reduction, photosynthesis, respiration, and so on [1]. It has been reported that Fe also attracts the interest of scientists due to its important role in the marine biogeochemical cycling process [2-4]. More importantly, Fe is also regarded as one of the growth-limiting factors for microorganisms and phytoplankton, which may have a relationship with the dominant phytoplankton species and red tide [5]. The biogeochemical effects of Fe depend largely on both its concentration and speciation [6]. In natural waters, Fe exists in different species and total Fe includes Fe ions $\left(\mathrm{Fe}^{2+}\right.$ and $\mathrm{Fe}^{3+}$ ), inorganically and organically complexed $\mathrm{Fe}$, colloidal $\mathrm{Fe}$, and particulate $\mathrm{Fe}$ [6]. Fe free ions and complexed $\mathrm{Fe}$ are differentiated as dissolved fraction and the others are particulate fraction. Acidification could release the unstable $\mathrm{Fe}$ by destroying the Fe-complexes and leaching the Fe in colloidal and particulate matters. The proportion of acidified dissolved $\mathrm{Fe}$ (ADFe) to total $\mathrm{Fe}$ is decided by the content and varieties of dissolved organic ligands in complex environmental water samples, and $\mathrm{ADFe}$ is more responsive to its bioavailability [7]. On the other hand, concentrations of ADFe in river water, coastal seawater and oceanic water are significantly different, and the biogeochemical process of $\mathrm{Fe}$ in coastal waters is much more complex owing to the terrigenous input and human activity [8]. Therefore, it is of significant to develop an accurate and simple method for the quantitative determination of ADFe in coastal waters.

Among a variety of analytical techniques for the determination of $\mathrm{Fe}$, the electrochemical cathodic stripping voltammetry (CSV) is recognized as a promising method with the advantages of high sensitivity, simple analytical processes, fast detection speed, portability, and potential for in situ or on-site detection [9]. In the past decades, mercury electrodes were widely adopted as working electrode for Fe determination with different complexing agents such as 2-(2-thiazolylazo)-p-cresol (TAC)

\footnotetext{
* Corresponding author.

E-mail addresses: dwpan@yic.ac.cn (D. Pan), zrden@hnu.edu.cn (R. Zhu).
} 
[10], 1-nitroso-2-naphthol (NN) [11], 2,3-dihydroxynaphthalene (DHN) [12], and others [13] added to decrease the detection limit (LOD). However, drawbacks of mercury in toxicity, storage and disposal make the mercury electrode limited in wide application [14]. In recent years, more and more chemically-modified electrodes have been developed for the voltammetric determination of $\mathrm{Fe}$ in different water samples [15-17]. However, considering the complex matrix conditions of coastal waters, continuous development of novel electrodes with high sensitivity and stability for the determination of Fe is still urgently required.

Recently, stainless steel micro-needle electrode (MNE) made of stainless steel acupuncture needle has been developed for electrochemical detection of different small biomolecules and inorganic ions due to their unique aciform structure, easy operation, high chemical stability, and large specific surface area [18-21]. MNE provides a promising electrochemical sensing platform due to its large specific surface area for modification of different functional nanomaterials and high hardness for potential in situ monitoring applications. Additionally, acupuncture is a traditional Chinese medical technique by inserting acupuncture needles into the body acupoints, and sometimes electric current is also applied. Therefore, chemical stability is a very important characteristic for acupuncture needles. So, the MNE made of stainless steel acupuncture needle also has the advantage of high chemical stability, which is exactly suitable for the electrochemical detection applications.

Nowadays, in order to improve the performance of the working electrode, nanomaterials have been widely adopted to fabricate the chemically modified electrodes [22,23]. As one of the star modification materials, gold nanomaterials have been successfully used for $\mathrm{Fe}$ determination with the advantages of high catalytic activity, good biocompatibility, and fast electron transfer rate [24-26]. However, the surface of stainless steel MNE is too smooth to load the gold nanomaterials firmly, so the surface modification of MNE is clearly needed [27]. Recently, German et al. described the formation of conducting polymers such as polyaniline, polypyrrole, polythiophene and their hybrid nanomaterials by enzymatic polymerization method [28,29]. Inorganic nanoparticles (e.g., $\mathrm{Au}$ or $\mathrm{Ag}$ nanoparticles) could be embedded into these conducting polymers with excellent properties such as high stability. Poly(3,4-ethylenedioxythiophene)-poly(sodium 4-styrenesulfonate) (PEDOT-PSS) is another conducting polymer which has been usually utilized for the surface modification of electrodes because of its outstanding conductivity and stability [30-32]. Some composites based on PEDOT-PSS and Au nanomaterials have been fabricated for sensing of different small biomolecules including NADH [33], caffeic acid [34], $\mathrm{H}_{2} \mathrm{O}_{2}$ [35], and tyramine [36] with excellent performance. Except for the improvement of conductivity, the PEDOT-PSS on MNE surface can provide more active sites for the combination of gold nanomaterials to improve the stability of the micro-needle sensor.

In this work, cluster-like gold nanomaterials (AuNCs) were immobilized on the conducting polymer PEDOT-PSS modified MNE to fabricate the novel electrochemical sensor (AuNCs/PEDOT-PSS/MNE) for the determination of $\mathrm{Fe}$ in coastal waters. The unique aciform structure of MNE provided larger specific surface area and the PEDOT-PSS provided the improved conductivity and more active sites for the combination and immobilization of AuNCs which had excellent catalytic activity towards the electrochemical reduction of $\mathrm{Fe}^{3+}$. The so-fabricated AuNCs/ PEDOT-PSS/MNE provided remarkably improved performance for the voltammetric determination of $\mathrm{Fe}^{3+}$. Additionally, the developed microneedle sensor was also successfully used for the determination of ADFe in a coastal river from the source to the estuary.

\section{Experimental}

\subsection{Reagents}

PSS (MW 70 000) and EDOT monomer ( $>97 \%$ ) were acquired from
Sigma-Aldrich. Chloroauric acid $\left(\mathrm{HAuCl}_{4} \cdot 4 \mathrm{H}_{2} \mathrm{O}\right)$ was purchased from Sinopharm Chemical Reagent Co. Ltd., China. The stock standard solution of Fe was supplied by Acros Organics. Buffer solutions with different $\mathrm{pH}$ were prepared with $0.1 \mathrm{M}$ acetic acid and sodium acetate, and adjusted with $\mathrm{HCl}$. All the reagents were analytical-grade chemicals and used without further purification unless otherwise stated. Stainless steel acupuncture needles purchased from Suzhou Medical Supplies Factory Co. Ltd (Suzhou, China) were used as the micro-needles to fabricate the MNEs. Silicone rubber obtained from Liyang Kangda Chemical Co. Ltd (China) was used as the insulating and sealing material. Deionized water (18.2 $\mathrm{M} \Omega \mathrm{cm}$ specific resistance) obtained from a Pall Cascada laboratory water system was used throughout the experiment.

\subsection{Apparatus}

The morphology characterization of different MNEs was conducted with the scanning electron microscopy (SEM, Hitachi S-4800 microscope, Japan). Energy dispersive X-ray spectroscopy (EDS HORIBAEX350, Japan) was used only for the element analysis. Electrochemical Work Station (CHI 660E, CH Instruments, Shanghai, China) was used for all of the electrochemical experiments. The developed AuNCs/PEDOT$\mathrm{PSS} / \mathrm{MNE}$ served as the working electrode, an $\mathrm{Ag} / \mathrm{AgCl}(3 \mathrm{M} \mathrm{KCl})$ was adopted as the reference electrode, and a platinum foil was used as the counter electrode. All potentials were measured with respect to the $\mathrm{Ag}$ / $\mathrm{AgCl}$ reference electrode.

\subsection{Preparation of the AuNCs/PEDOT-PSS/MNE}

Scheme 1 shows the fabrication process of the AuNCs/PEDOT-PSS/ MNE for the detection of $\mathrm{Fe}^{3+}$ in coastal waters. Firstly, the MNE was prepared according to our previous work with minor modification [20]. Simply, the stainless steel needle was covered with the silicone rubber which acted as insulating and sealing material, and only the needle tip (about $1-2 \mathrm{~mm}$ ) was left as the sensing surface and the needle handle as the electrode connection. Then, the surface modification of MNE with PEDOT-PSS (PEDOT-PSS/MNE) was achieved by cycling the potential between -0.8 and $1.3 \mathrm{~V}$ in the aqueous solution with $0.01 \mathrm{M}$ EDOT and PSS for 60 circles at the scan rate of $50 \mathrm{mV} / \mathrm{s}$. Lastly, the AuNCs/PEDOT-PSS/MNE was prepared through potentiostatic deposition method in $2 \mathrm{mM} \mathrm{HAuCl}_{4}$ solution with the deposition potential and time of $-0.3 \mathrm{~V}$ and $120 \mathrm{~s}$, respectively. The AuNCs/PEDOT-PSS/MNE was obtained eventually after the cleaning and drying processes at room temperature.

\subsection{Preparation of coastal water samples}

Coastal water samples were collected from the Guangdang River which flows into the Northern Yellow Sea, Shandong Province, China. A total of 19 samples were collected from the source to the estuary. All the water samples were adjusted to $\mathrm{pH} 1.8$ with $\mathrm{HCl}$, stored in acid-cleaned polyethylene bottles, filtrated with $0.45 \mu \mathrm{m}$ membranes, and kept in the fridge $\left(4^{\circ} \mathrm{C}\right)$ until determination. The samples were diluted five times with buffer solution ( $\mathrm{pH} 2.0$ ), determined with differential pulse voltammetry (DPV), and quantified with standard addition method.

\subsection{Electrochemical analysis procedure}

Electrochemical properties of different modified MNEs were characterized by cyclic voltammetry (CV) in buffer solution ( $\mathrm{pH} 3.5)$ in the range of -0.4 to $0.6 \mathrm{~V}$ and $0.2-1.3 \mathrm{~V}$, respectively. Unless otherwise stated, the voltammetric responses of $\mathrm{Fe}^{3+}$ were obtained with DPV in buffer solution ( $\mathrm{pH}$ 2.0). The DPV scans were carried out using the following parameters: initial potential of $0.8 \mathrm{~V}$, final potential of $0.2 \mathrm{~V}$, amplitude of $0.05 \mathrm{~V}$, potential incremental of $0.004 \mathrm{~V}$, pulse width of $0.05 \mathrm{~s}$, pulse period of $0.5 \mathrm{~s}$, and quiet time of $2 \mathrm{~s}$, respectively. 


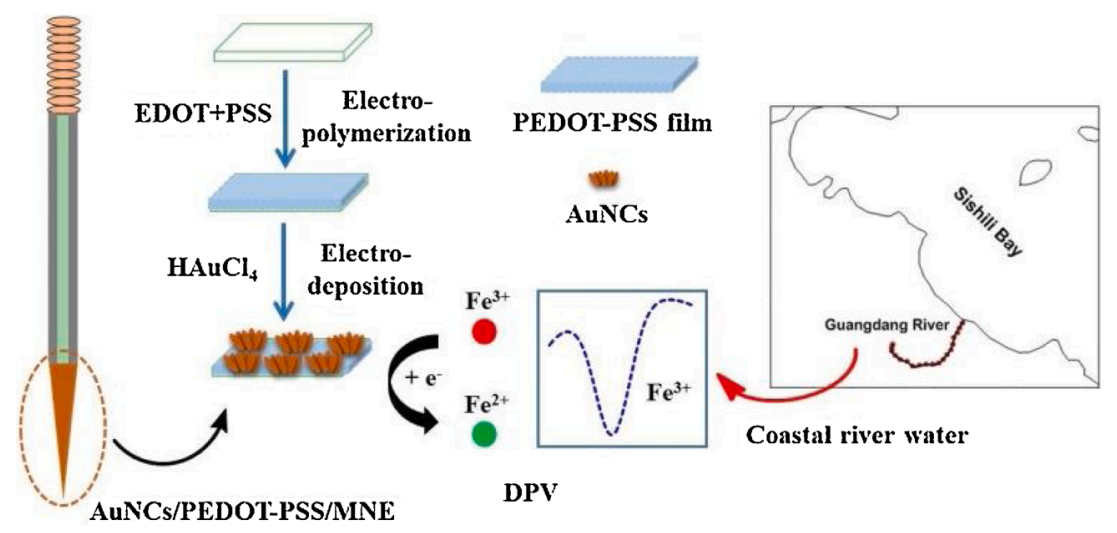

Scheme 1. Schematic illustration for the fabrication process of AuNCs/PEDOT-PSS/MNE and voltammetric detection of Fe ${ }^{3+}$ in coastal waters.

\section{Results and discussion}

\subsection{Characterization of the AuNCs/PEDOT-PSS/MNE}

After fabrication, the surface morphology of the AuNCs/PEDOT-PSS/ MNE was characterized firstly by SEM (Fig. 1). It had been exhibited and discussed in our previous works that the surface of MNE was very smooth with limited binding sites for functional nanomaterials [20,21, 27]. As to the PEDOT-PSS/MNE (Fig. 1A), after the modification of PEDOT-PSS, there was a film covered on the surface of MNE which made the surface more rough. As a conducting polymer film, the PEDOT-PSS improved the conductivity and specific surface area of the electrode and provided more active sites for the combination of gold nanomaterials. It could be seen from Fig. 1B, C that the AuNCs composed of gold nanobranches were immobilized on the electrode surface by the film-like PEDOT-PSS. The cluster-like structure of AuNCs might be caused by the PEDOT-PSS polymer on MNE surface which acted as the morphology-controlling agent partially. As exhibited in our previous works that $\mathrm{C}, \mathrm{Fe}$ and $\mathrm{Cr}$ were the main elements of the so-fabricated MNE $[21,27,37]$. Considering its high chemical stability, the element composition of MNE had no effect on the electrochemical determination of metal ions. It could be seen from the EDS pattern of AuNCs/PEDOT-PSS/MNE (Fig. 1D) that the typical Au peaks appeared at about $1.64,2.15$, and $9.71 \mathrm{KeV}$, which proved that the cluster-like nanomaterials were AuNCs exactly.

The electrochemical properties of the so-fabricated AuNCs/PEDOTPSS/MNE were investigated by CV in buffer solution (0.1 M, pH 3.5). Fig. 2A showed the typical cyclic voltammograms of the bare MNE, PEDOT-PSS/MNE, and AuNCs/PEDOT-PSS/MNE in the range of - 0.4 to $0.6 \mathrm{~V}$. It could be observed that the background current of the MNE was significantly enhanced after the surface modification of PEDOT-PSS, which indicated the improved conductivity of the PEDOT-PSS/MNE. As expected, the background current of AuNCs/PEDOT-PSS/MNE was further enhanced after the modification of AuNCs due to its excellent performance in facilitating electron transfer. Fig. 2B showed the cyclic voltammogram of AuNCs/PEDOT-PSS/MNE in buffer solution from 0.2 to $1.3 \mathrm{~V}$. There appeared a typical peak of $\mathrm{Au}^{0}$ at about $0.8 \mathrm{~V}$, which further proved the presence of AuNCs.

Electrochemical impedance spectroscopy (EIS) was also applied to verify the effects of AuNCs and PEDOT-PSS on the electron transfer
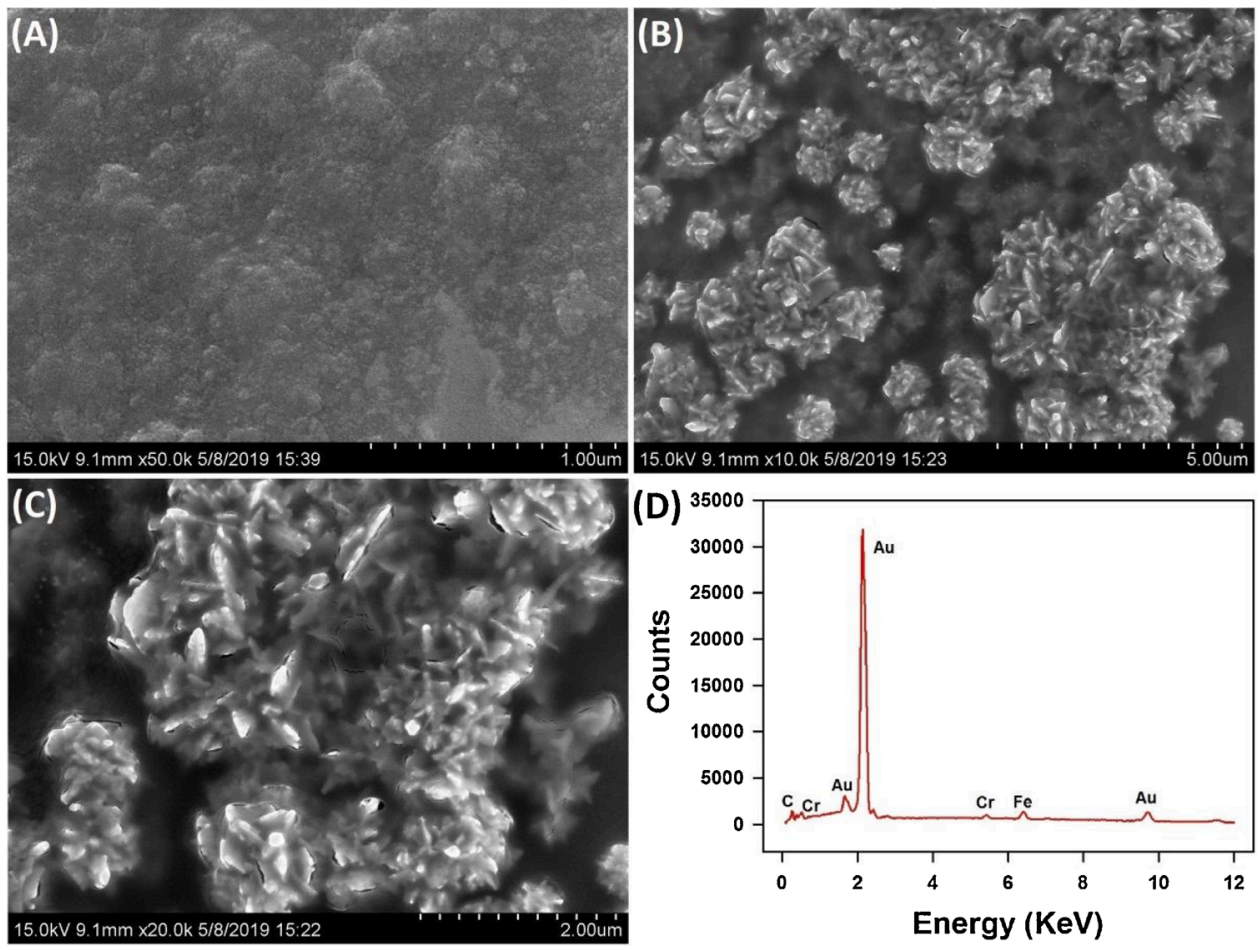

Fig. 1. SEM images of the PEDOT-PSS/MNE (A), AuNCs/PEDOT-PSS/MNE (B, C) and EDS pattern of the AuNCs/PEDOT-PSS/MNE (D). 

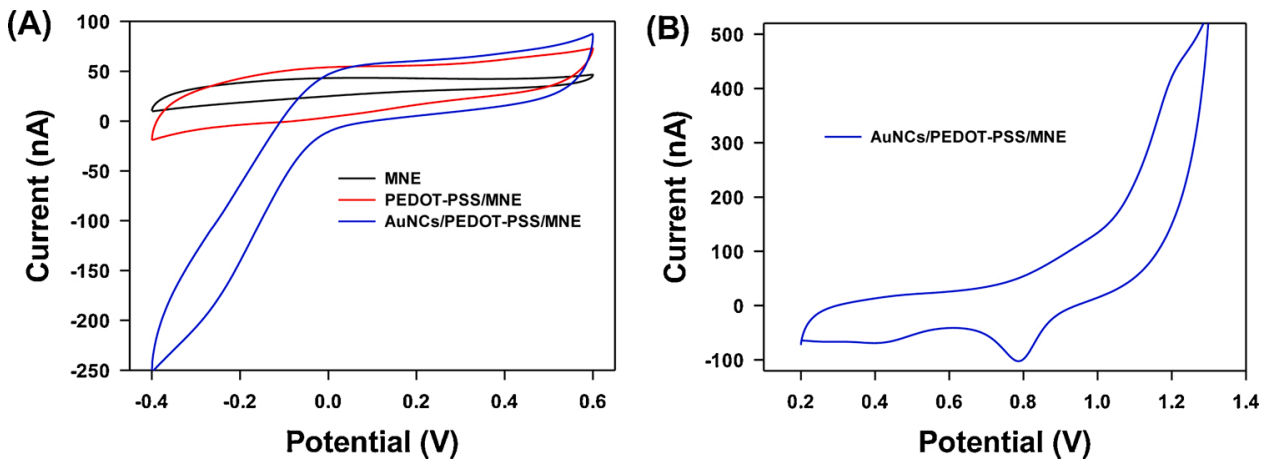

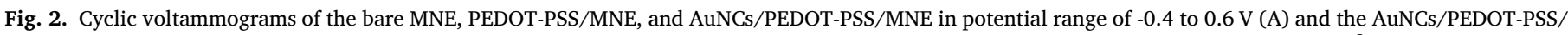
MNE in potential range of 0.2 to $1.3 \mathrm{~V}$ (B) in buffer solution $(0.1 \mathrm{M}, \mathrm{pH} 3.5)$ with the scan rate of $50 \mathrm{mV} / \mathrm{s}$. The electrode area was $0.4 \mathrm{~mm}$.

capability. Fig. 3 showed the Nyquist plots and equivalent circuits of the MNE, PEDOT-PSS/MNE, and AuNCs/PEDOT-PSS/MNE using [Fe $\left.(\mathrm{CN})_{6}\right]^{3-} /\left[\mathrm{Fe}(\mathrm{CN})_{6}\right]^{4-}$ redox couple as the electrochemical probe. The results of Nyquist plots (Fig. 3A) of different MNEs could be interpreted by the corresponding equivalent circuits (Fig. 3B), which included four circuit elements: the resistance of solution $\left(R_{S}\right)$, the double layer capacitance $\left(\mathrm{C}_{\mathrm{dl}}\right)$, the charge transfer resistance $\left(\mathrm{R}_{\mathrm{ct}}\right)$, and the Warburg impedance $\left(Z_{W}\right)$ [38]. As reported, the $R_{c t}$ can be affected by the electrode fabrication and detection process [38]. So, the circuit element $\mathrm{R}_{\mathrm{ct}}$ was especially concerned in this work. The Nyquist plot of bare MNE could be interpreted by the standard Randles equivalent circuit (equivalent circuit a). This circuit has also been used for the interpretation of the Nyquist plot of bare graphite electrode as reported previously [39]. The $R_{\mathrm{ct}}$ of bare MNE was calculated as $40.2 \mathrm{M} \Omega$, which confirmed the slow process of charge transfer on the bare MNE. After the modification of MNE surface with PEDOT-PSS, the recorded EIS shape differed from that of the bare MNE, and the corresponding equivalent circuit was characterized with another RC part (equivalent circuit b). Although the polymers modified electrodes could also be interpreted roughly by the circuit a $[27,38]$, circuit $b$ fit more well with the experimental results. The similar results have been obtained in the researches about the 1,10-Phenanthroline-5,6-dione and polypyrrole film deposited electrodes $[39,40]$. After the modification of PEDOT-PSS, the $\mathrm{R}_{\mathrm{ct}}$ of the electrode was decreased remarkably to $2.6 \mathrm{M} \Omega$. The change was mostly related to the property of PEDOT-PSS, which showed that the PEDOT-PSS was a good conductor, what was a useful characteristic for electron transfer mediator [39]. The semicircle diameter of the Nyquist plot at high frequency part can also reflect the $\mathrm{R}_{\mathrm{ct}}$. It could be concluded from Fig. 3A that the semicircle diameter of PEDOT-PSS/MNE was much less than that of MNE, which was consistent with the calculation results. The Nyquist plot of AuNCs/PEDOT-PSS/MNE could also be interpreted by the equivalent circuit $\mathrm{b}$ model. As expected, after the modification of AuNCs, the $\mathrm{R}_{\mathrm{ct}}$ of AuNCs/PEDOT-PSS/MNE further decreased to $1.2 \mathrm{M} \Omega$, which showed the excellent property of AuNCs in promoting electron transfer. It should be noted that the $\mathrm{R}_{\mathrm{ct}}$ values of the MNE, PEDOT-PSS/MNE, and AuNCs/PEDOT-PSS/MNE were all higher than that of the bare and 1,10-Phenanthroline-5,6-dione modified graphite electrodes [39]. The relative poor electro-conductivity of stainless steel MNE might be the explanation of the phenomenon. The excellent electrochemical properties of AuNCs and PEDOT-PSS were very helpful for the voltammetric determination of $\mathrm{Fe}$ with the AuNCs/PEDOT-PSS/MNE. Further, the electrode kinetics was also studied in $\mathrm{K}_{3}\left[\mathrm{Fe}(\mathrm{CN})_{6}\right]$ solution via $\mathrm{CV}$ with variable scan rates. The current responses of $\mathrm{K}_{3}\left[\mathrm{Fe}(\mathrm{CN})_{6}\right]$ obtained on AuNCs/PEDOT-PSS/MNE had a linear relationship with the square root of the scan rates, which was similar with our previous work [20]. Therefore, the redox reaction of $\mathrm{K}_{3}\left[\mathrm{Fe}(\mathrm{CN})_{6}\right]$ on the AuNCs/PEDOT-PSS/MNE was a diffusion-controlled electrode process.

\subsection{Electrochemical response of AuNCs/PEDOT-PSS/MNE to $\mathrm{Fe}^{3+}$}

The voltammetric responses of $\mathrm{Fe}^{3+}$ on different modified MNEs were investigated by using DPV (Fig. 4). In the determination of $\mathrm{Fe}^{3+}$ by $\mathrm{CSV}, \mathrm{Fe}^{3+}$ was adsorbed on the surface of the electrode and combined with the electron $\left(\mathrm{e}^{-}\right)$to be reduced to $\mathrm{Fe}^{2+}$, and the corresponding reduction peak was recorded in the voltammetric diagram. It was clear that no reduction current signal of $\mathrm{Fe}^{3+}$ could be observed on the bare MNE and PEDOT-PSS/MNE. So, although the modification of PEDOTPSS improved the conductivity of the electrode, it had almost no contribution to the improvement of the voltammetric response of $\mathrm{Fe}^{3+}$. When the AuNCs/PEDOT-PSS/MNE was concerned, a large current signal induced by the reduction of $\mathrm{Fe}^{3+}$ to $\mathrm{Fe}^{2+}$ appeared at about

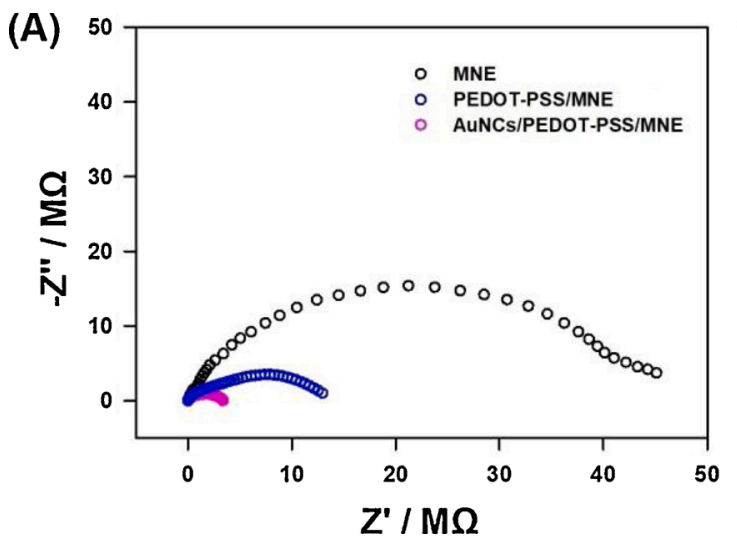

(B)
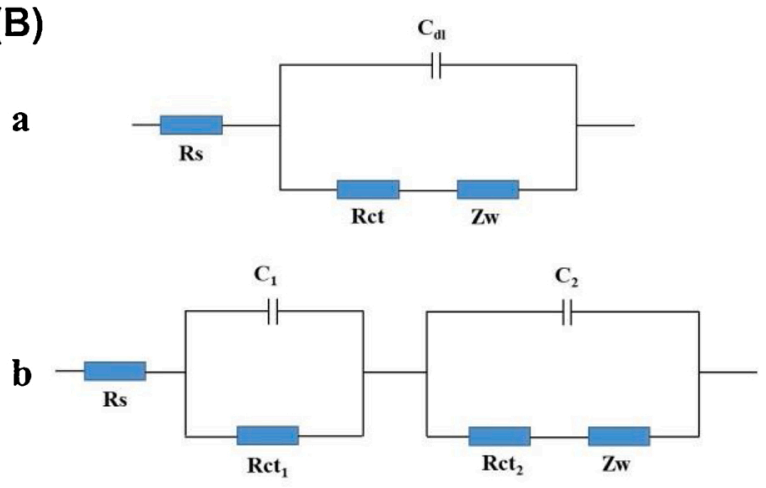

Fig. 3. Nyquist plots (A) and equivalent circuits (B) of the bare MNE, PEDOT-PSS/MNE, and AuNCs/PEDOT-PSS/MNE in $0.1 \mathrm{M} \mathrm{KCl} \mathrm{solution} \mathrm{containing} 5 \mathrm{mM} \mathrm{K}$ [ $[\mathrm{Fe}$ $\left.(\mathrm{CN})_{6}\right]$. The EIS were recorded in the frequency range from $0.01 \mathrm{~Hz}$ to $100 \mathrm{kHz}$ at the potential of $0.2 \mathrm{~V} v s . \mathrm{Ag} / \mathrm{AgCl}$ (excitation amplitude, $50 \mathrm{mV}$ ). 


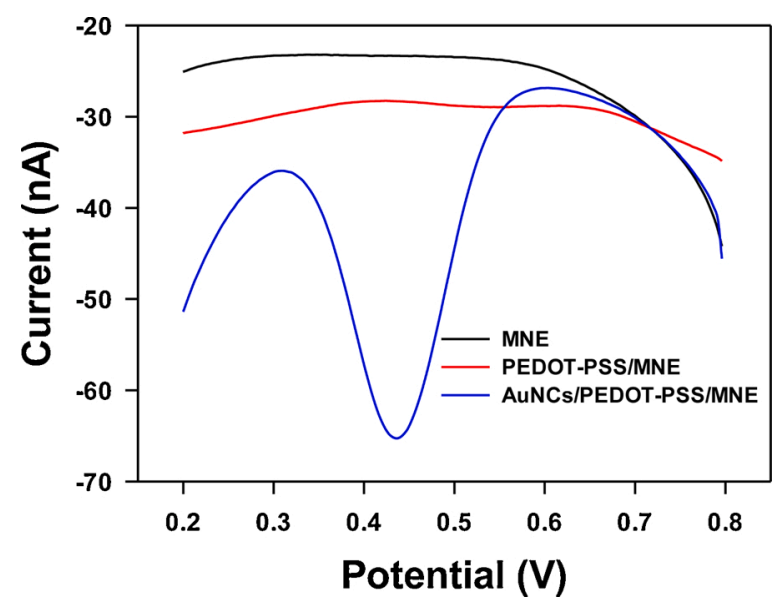

Fig. 4. Differential pulse voltammograms of the bare MNE, PEDOT-PSS/MNE, and AuNCs/PEDOT-PSS/MNE for $3 \mu \mathrm{M} \mathrm{Fe}^{3+}$. Electrode area, $0.4 \mathrm{~mm}^{2}$; scan rate, $8 \mathrm{mV} / \mathrm{s}$.

$0.43 \mathrm{~V}$, indicating the excellent electrocatalytic performance of AuNCs for the voltammetric determination of $\mathrm{Fe}^{3+}$. It could be concluded from the comparison of different MNEs for $\mathrm{Fe}^{3+}$ determination that the AuNCs/PEDOT-PSS/MNE showed an enhanced performance by combining the excellent properties of PEDOT-PSS and AuNCs.

\subsection{Optimization for $\mathrm{Fe}^{3+}$ determination with the AuNCs/PEDOT-PSS/} MNE

The effects of experimental parameters of $\mathrm{pH}$ value and electrodeposition time of AuNCs on the voltammetric response of $\mathrm{Fe}^{3+}$ on the AuNCs/PEDOT-PSS/MNE were also studied. To investigate the influence of $\mathrm{pH}$ value of buffer solution to the current response of $5 \mu \mathrm{M} \mathrm{Fe}^{3+}$, different $\mathrm{pH}$ values from 1.5 to 3.5 were applied during the determination process (Fig. 5A). The results demonstrated that the maximum peak current (Ip) of $\mathrm{Fe}^{3+}$ reduction was obtained at $\mathrm{pH} 2.0$ and the Ip decreased gradually with the $\mathrm{pH}$ increases from 2.0 to 3.5. Considering the hydrolysis of $\mathrm{Fe}^{3+}$ in a slightly alkaline environment, this phenomenon could be easily understood, and similar results have been reported in the previous literature [41]. So, $\mathrm{pH} 2.0$ was elected and adopted as the optimal $\mathrm{pH}$ value in this work.

The effect of deposition time of AuNCs was investigated in the range of 60 to $360 \mathrm{~s}$. As shown in Fig. 5B, the Ip gradually increased with deposition time from 60 to $180 \mathrm{~s}$, and then decreased from 180 to $360 \mathrm{~s}$. It has been illustrated that the AuNCs with cluster-like structure exhibited excellent electrocatalytic performance for the voltammetric detection of $\mathrm{Fe}^{3+}$. From 60 to $180 \mathrm{~s}$, more gold nanobranches deposited on the electrode surface to form the cluster-like structure, and the Ip increased accordingly. However, when the deposition time increased continuously to $360 \mathrm{~s}$, the cluster-like structure began to be destroyed and the electroactive area decreased due to the aggregation of gold nanomaterials. Therefore, $180 \mathrm{~s}$ was adopted as the deposition time of AuNCs in this work.

\subsection{Calibration curve}

Subsequently, the analytical performance of the micro-needle sensor including linear range and detection limit (LOD) was investigated. The calibration curve for $\mathrm{Fe}^{3+}$ determination was derived from the differential pulse voltammograms obtained on AuNCs/PEDOT-PSS/MNE under the optimal conditions described above (Fig. 6). It could be seen that the Ip was linear with $\mathrm{Fe}^{3+}$ concentration in the range of $0.01-5 \mu \mathrm{M}$ with the linear regression equation $I p=11.48 C+0.42$ (0.998). The LOD of AuNCs/PEDOT-PSS/MNE for the voltammetric determination of $\mathrm{Fe}^{3+}$ was calculated as $3.1 \mathrm{nM}(\mathrm{s} / \mathrm{n}=3)$. Table 1 showed the comparison of AuNCs/PEDOT-PSS/MNE with the others proposed previously in the literatures for $\mathrm{Fe}^{3+}$ determination. It could be concluded that the sofabricated AuNCs/PEDOT-PSS/MNE might be a good candidate for the voltammetric determination of $\mathrm{Fe}$ with satisfactory performance, no additional reagents added, low price, and simple fabrication process.

\subsection{Reproducibility, repeatability and selectivity}

The reproducibility of the AuNCs/PEDOT-PSS/MNE for Fe determination was investigated in $3 \mu \mathrm{M} \mathrm{Fe}{ }^{3+}$ utilizing six independently fabricated micro-needle sensor with the same method, and the relative

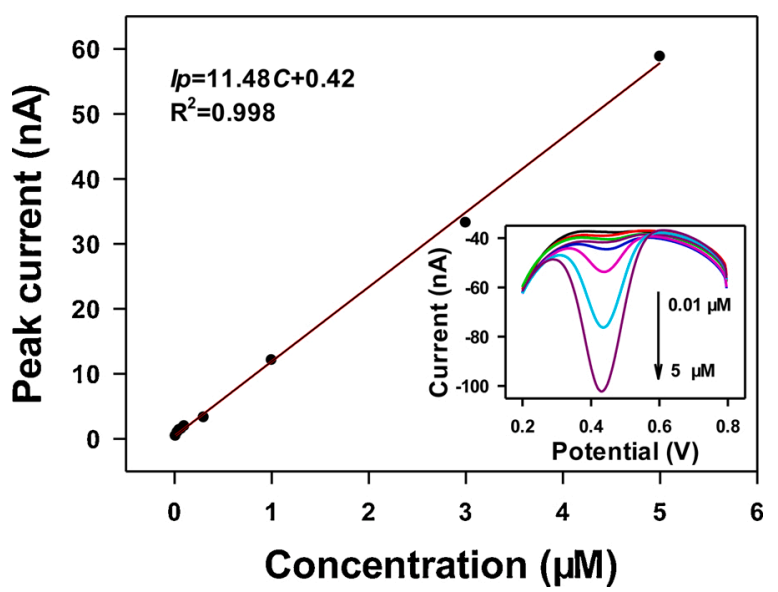

Fig. 6. Calibration curve of $\mathrm{Fe}^{3+}$ on the AuNCs/PEDOT-PSS/MNE with concentration from 0.01 to $5 \mu \mathrm{M}$ and the corresponding differential pulse voltammograms obtained in buffer solution $(0.1 \mathrm{M}, \mathrm{pH} 2.0)$ with successive addition of $0.01,0.03,0.05,0.07,0.1,0.3,1,3,5 \mu \mathrm{M} \mathrm{Fe}^{3+}$. Electrode area, $0.4 \mathrm{~mm}^{2}$; scan rate, $8 \mathrm{mV} / \mathrm{s}$.
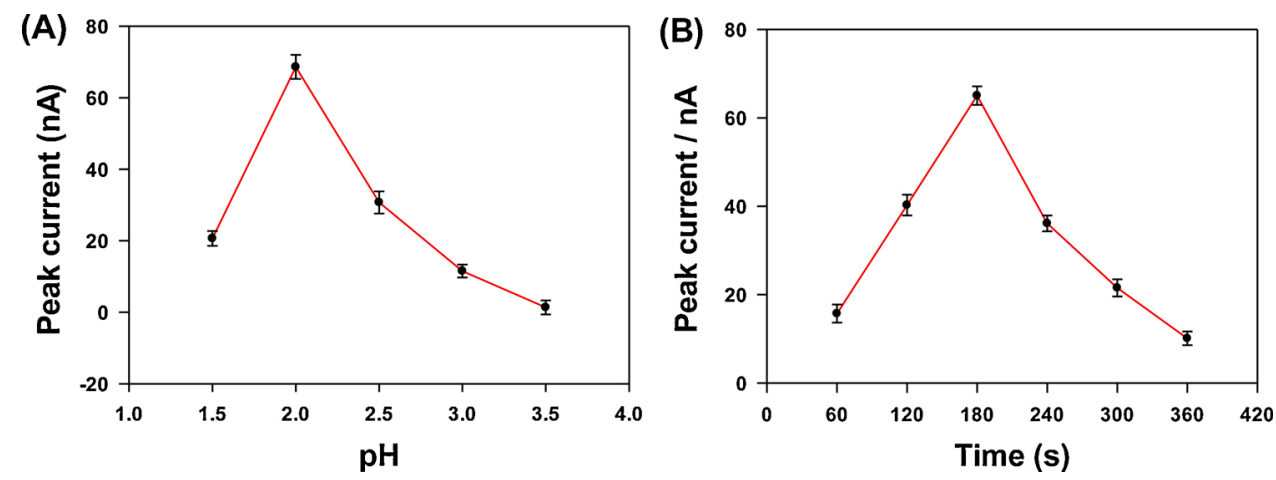

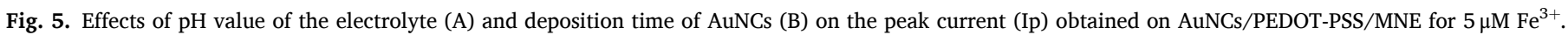


Table 1

Comparison of the AuNCs/PEDOT-PSS/MNE with other electrodes for $\mathrm{Fe}^{3+}$ determination.

\begin{tabular}{|c|c|c|c|c|c|c|}
\hline Technique $^{\mathrm{a}}$ & Electrode $^{\mathrm{b}}$ & Modifier $^{c}$ & Reagents $^{\mathrm{d}}$ & Linear range $(\mu \mathrm{M})$ & Detection limit (nM) & Ref. \\
\hline DPV & DM/HMDE & No & Tri-BAP & $0.5-50$ & 1500 & [42] \\
\hline AdCSV & HMDE & No & SVRS & $0.09-3.58$ & 32 & [43] \\
\hline AdCSV & HMDE & No & DMG, Catechol & - & 1.3 & [44] \\
\hline CSV & GCE & Chitosan, oxalates & No & $0.36-5.4$ & 180 & [45] \\
\hline SWV & GCE & IL-rGO/AuNDs/Nafion & No & $0.3-100$ & 35 & {$[25]$} \\
\hline SWV & GCE & nano-TiC, Nafion & No & $0.07-70$ & 7.2 & [46] \\
\hline DPV & GCE & rGO/AuNPs & 5-Br-PADAP & $0.03-3$ & 3.5 & [26] \\
\hline DPV & GCE & BiNS & No & $0.01-20$ & 2.3 & [17] \\
\hline FIA & GCE & MWCNTs, PtNPs & No & $0-10,000$ & 3.0 & [47] \\
\hline sWV & CPE & No & 5-Br-PADAP & $0.006-0.45$ & 1.7 & [48] \\
\hline DPV & MNE & AuNCs/PEDOT-PSS & No & $0.01-5$ & 3.1 & This work \\
\hline
\end{tabular}

${ }^{a}$ Technique: AdCSV, adsorptive cathodic stripping voltammetry; CSV, cathodic stripping voltammetry; SWV, square wave voltammetry; FIA, flow injection analysis.

b Electrode: DME, drop mercury electrode; HMDE, hanging mercury drop electrode; GCE, glassy carbon electrode; CPE, carbon paste electrode.

c Modifier: IL-rGO/AuNDs/Nafion, ionic liquid-reduced graphene oxide/gold nanodendrites/Nafion; nano-TiC, titanium carbide nanoparticles; rGO/AuNPs, reduced graphene oxide/gold nanoparticles; BiNS, bismuth nanosheets; MWCNTs, multiwall carbon nanotubes; PtNPs, Pt nanoparticles.

d Agent: Tri-BAP, tributylammonium perchlorate; SVRS, Solochrome Violet RS; DMG, dimethylglyoxime; 5-Br-PADAP, 2-(5-bromo-2-pyridylazo)-5diethylaminophenol.

standard deviation (RSD) was $5.4 \%$. The repeatability of the developed method was studied by detecting $3 \mu \mathrm{M} \mathrm{Fe}^{3+}$ with the same AuNCs/ PEDOT-PSS/MNE for six measurements, and the RSD was calculated as $3.7 \%$. So, the AuNCs/PEDOT-PSS/MNE showed a good reproducibility and repeatability towards the voltammetric determination of Fe. Additionally, the AuNCs/PEDOT-PSS/MNE was used for five measurements in each day and stored in air condition. The results showed that the current response had no obvious decrease during six days, which reflected a good stability of the AuNCs/PEDOT-PSS/MNE.

To investigate the suitability of the AuNCs/PEDOT-PSS/MNE for Fe determination in real coastal waters, the selectivity was verified by adding different foreign species into the buffer solution with $3 \mu \mathrm{M} \mathrm{Fe}^{3+}$. The results showed that 100 -fold $\mathrm{Ca}^{2+}, 50$-fold $\mathrm{Mg}^{2+}, \mathrm{Mn}^{2+}, 30$-fold $\mathrm{Cr}^{3+}, 10$-fold $\mathrm{Zn}^{2+}, \mathrm{Pb}^{2+}, \mathrm{Cu}^{2+}$ and $\mathrm{Cd}^{2+}$ had no effect on the voltammetric determination of $\mathrm{Fe}^{3+}$ ( $<5 \%$ of current change). Thus, the newly fabricated AuNCs/PEDOT-PSS/MNE had a promising potential for $\mathrm{Fe}$ determination in coastal water samples with a good anti-interference ability.

\subsection{Determination of Fe in real coastal river water}

The practical analytical application of the AuNCs/PEDOT-PSS/MNE was illustrated by the determination of ADFe in a real coastal river, the Guangdang River which flows into Sishili Bay, Northern Yellow Sea, China. The coastal river water samples were diluted five times and quantified by the standard addition method. Fig. 7A showed the typical differential pulse voltammograms obtained on the AuNCs/PEDOT-PSS/ MNE in a river water sample with the successive addition of $0,1,2$, and

$4 \mu \mathrm{M} \mathrm{Fe}^{3+}$ and the corresponding linear regression curve. The $\mathrm{Fe}^{3+}$ concentration in this coastal river water sample was calculated to be $2.22 \mu \mathrm{M}$. The results of determination of total Fe in coastal water samples by AuNCs/PEDOT-PSS/MNE and atomic absorption spectrometry (AAS) were shown in Table 2. It could be seen that the results obtained on AuNCs/PEDOT-PSS/MNE were consistent with AAS, which indicated that the fabricated AuNCs/PEDOT-PSS/MNE might be a promising electrochemical sensor for Fe determination in coastal waters.

Subsequently, the AuNCs/PEDOT-PSS/MNE was used for the determination of ADFe in the whole Guangdang River. A total of 19 samples from the source to the estuary were collected and determined, and the corresponding concentrations of $\mathrm{ADFe}$ were presented in Fig. 7B. It could be seen that the concentration of ADFe in Guangdang River was relatively high ranging from 1.5 to $10.0 \mu \mathrm{M}$. The concentrations of ADFe in samples G3, G9, G11, G13, and G15 ( $8.0 \mu \mathrm{M}$ on average) were relatively higher than the others ( $3.0 \mu \mathrm{M}$ on average). Sampling sites of these 5 samples were close to the residential areas and human activities might be the explanation for increase of ADFe contents. The change of salinity of samples had almost no effect on the detection of Fe with the AuNCs/

Table 2

Comparison of the AuNCs/PEDOT-PSS/MNE and AAS for determination of total Fe in coastal water samples $(n=3)$.

\begin{tabular}{lll}
\hline Samples & Detected by this sensor $(\mu \mathrm{M})$ & Detected by AAS $(\mu \mathrm{M})$ \\
\hline Coastal water 1 & $11.12 \pm 0.15$ & 11.32 \\
Coastal water 2 & $15.70 \pm 0.15$ & 15.68 \\
Coastal water 3 & $26.48 \pm 0.30$ & 26.09 \\
\hline
\end{tabular}
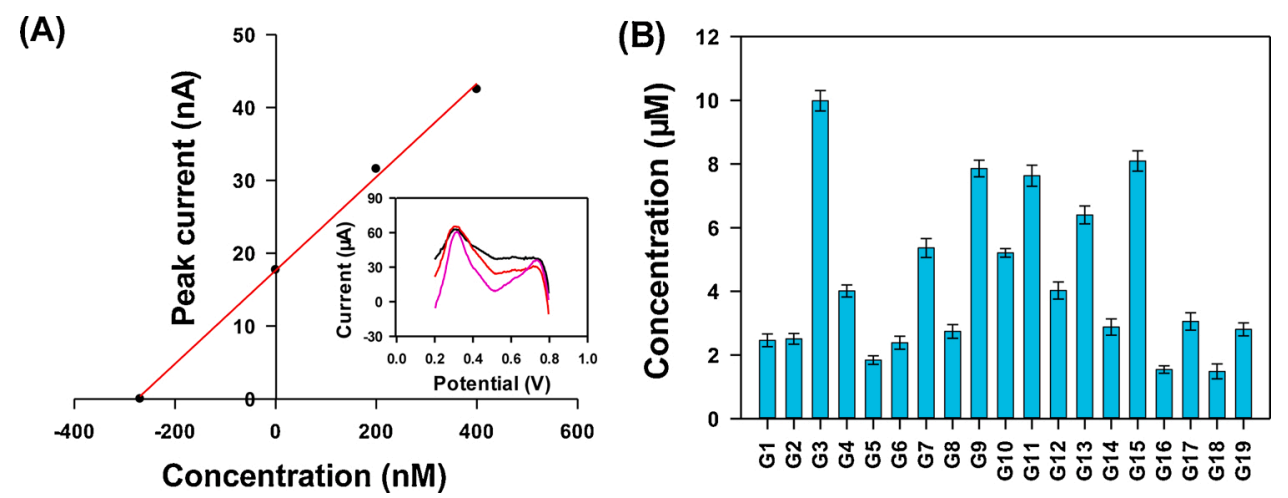

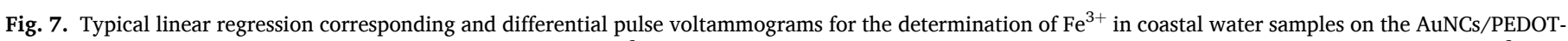

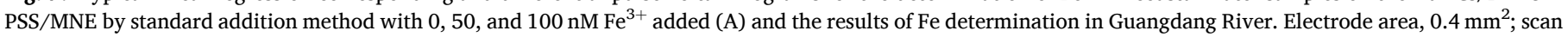
rate, $8 \mathrm{mV} / \mathrm{s}$. 
PEDOT-PSS/MNE proposed in this work. The results demonstrated that the AuNCs/PEDOT-PSS/MNE fabricated here was exactly suitable for the determination of $\mathrm{Fe}$ in coastal river water.

\section{Conclusions}

In summary, a novel micro-needle sensor based on AuNCs immobilized with PEDOT-PSS was developed for the determination of $\mathrm{Fe}$ in coastal waters. The combined effects of the unique structure of MNE, the good conductivity of PEDOT-PSS, and excellent catalytic properties of AuNCs made the AuNCs/PEDOT-PSS/MNE a promising sensor for the determination of ADFe in coastal river water, which was of great significance for monitoring the coastal environment. Furthermore, the determination of other metal elements in different environmental water samples could also be studied by the micro-needle sensor based on different functional nanomaterials in the future.

\section{CRediT authorship contribution statement}

Haitao Han: Conceptualization, Methodology, Writing - original draft. Dawei Pan: Conceptualization, Writing - review \& editing. Fei Pan: Methodology. Xueping Hu: Methodology. Rilong Zhu: Writing review \& editing.

\section{Declaration of Competing Interest}

The authors declare that they have no known competing financial interests or personal relationships that could have appeared to influence the work reported in this paper.

\section{Acknowledgments}

This work was financially supported by the Original Innovation Project of Chinese Academy of Sciences (ZDBS-LY-DQC009), the Strategic Priority Research Program of the Chinese Academy of Sciences (XDB42000000), the National Key R\&D Program of China (2019YFD0901103), the National Natural Science Foundation of China (21906075), the Key Research and Development Plan of Yantai City (2018ZHGY076), and the Shandong Key Laboratory of Coastal Environmental Processes, YICCAS (2019SDHADKFJJ14).

\section{References}

[1] W.G. Sunda, S.A. Huntsman, Iron uptake and growth limitation in oceanic and coastal phytoplankton, Mar. Chem. 50 (1995) 189-206.

[2] P.J. Statham, Y. Jacobson, C.M.G. van den Berg, The measurement of organically complexed $\mathrm{Fe}^{\mathrm{II}}$ in natural waters using competitive ligand reverse titration, Anal. Chim. Acta 743 (2012) 111-116.

[3] Z. Chase, K.S. Johnson, V.A. Elrod, J.N. Plant, S.E. Fitzwater, L. Pickell, C M. Sakamoto, Manganese and iron distributions off central California influenced by upwelling and shelf width, Mar. Chem. 95 (2005) 235-254.

[4] B. D'Autreaux, N.P. Tucker, R. Dixon, S. Spiro, A non-haem iron centre in the transcription factor NorR senses nitric oxide, Nature 437 (2005) 769-772.

[5] M.L. Wells, C.G. Trick, Controlling iron availability to phytoplankton in ironreplete coastal waters, Mar. Chem. 86 (2004) 1-13.

[6] J. Guan, B. Yan, H. Zhu, L. Wang, D. Lu, L. Cheng, Flux characteristics of total dissolved iron and its species during extreme rainfall event in the midstream of the Heilongjiang River, J. Environ. Sci. 30 (2015) 74-80.

[7] Y. Zhu, X. Hu, D. Pan, H. Han, M. Lin, Y. Lu, C. Wang, R. Zhu, Speciation determination of iron and its spatial and seasonal distribution in coastal river, Sci. Rep. 8 (2018) 2576.

[8] E.P. Achterberg, T.W. Holland, A.R. Bowie, R.F.C. Mantoura, P.J. Worsfold, Determination of iron in seawater, Anal. Chim. Acta 442 (2001) 1-14.

[9] D. Pan, Y. Wang, Z. Chen, T. Lou, W. Qin, Nanomaterial/ionophore-based electrode for anodic stripping voltammetric determination of lead: an electrochemical sensing platform toward heavy metals, Anal. Chem. 81 (2009) 5088-5094.

[10] P.L. Croot, M. Johansson, Determination of iron speciation by vathodic dtripping voltammetry in seawater using the competing ligand 2-(2-Thiazolylazo)-p-cresol (TAC), Electroanalysis 12 (2000) 565-576.

[11] M. Cheize, G. Sarthou, P.L. Croot, E. Bucciarelli, A.C. Baudoux, A.R. Baker, Iron organic speciation determination in rainwater using cathodic stripping voltammetry, Anal. Chim. Acta 736 (2012) 45-54.
[12] H. Obata, C.M.G. van den Berg, Determination of picomolar levels of iron in seawater using catalytic cathodic stripping voltammetry, Anal. Chem. 73 (2001) 2522-2528.

[13] M. Lu, N.V. Rees, A.S. Kabakaev, R.G. Compton, Determination of iron: electrochemical methods, Electroanalysis 24 (2012) 1693-1702.

[14] J. Wang, Stripping analysis at bismuth electrodes: a review, Electroanalysis 17 (2005) 1341-1346.

[15] E.A. Zakharova, E.E. Elesova, G.N. Noskova, M. Lu, R.G. Compton, Direct voltammetric determination of total iron with a gold microelectrode Ensemble, Electroanalysis 24 (2012) 2061-2069.

[16] H. Han, D. Pan, C. Wang, R. Zhu, Controlled synthesis of dendritic gold nanostructures by graphene oxide and their morphology-dependent performance for iron detection in coastal waters, RSC Adv. 7 (2017) 15833-15841.

[17] X. Hu, D. Pan, M. Lin, H. Han, F. Li, Graphene oxide-assisted synthesis of bismuth nanosheets for catalytic stripping voltammetric determination of iron in coastal waters, Microchim. Acta 183 (2015) 855-861.

[18] J.X. Zhou, L.N. Tang, F. Yang, F.X. Liang, H. Wang, Y.T. Li, G.J. Zhang, MoS $2 / \mathrm{Pt}$ nanocomposite-functionalized microneedle for real-time monitoring of hydrogen peroxide release from living cells, Analyst 142 (2017) 4322-4329.

[19] X. Niu, Z. Wen, X. Li, W. Zhao, X. Li, Y. Huang, Q. Li, G. Li, W. Sun, Fabrication of graphene and gold nanoparticle modified acupunctureneedle electrode and its application in rutin analysis, Sens. Actuators B Chem. 255 (2018) 471-477.

[20] H. Han, W. Tao, X. Hu, X. Ding, D. Pan, C. Wang, S. Xu, Needle-shaped electrode for speciation analysis of copper in seawater, Electrochim. Acta 289 (2018) 474-482.

[21] Y. Li, H. Han, D. Pan, P. Zhang, Fabrication of a micro-needle sensor based on copper microspheres and polyaniline film for nitrate determination in coastal river waters, J. Electrochem. Soc. 166 (2019) B1038-B1043.

[22] I. Khalil, S. Rahmati, N. Muhd Julkapli, W.A. Yehye, Graphene metal nanocomposites - recent progress in electrochemical biosensing applications, J. Ind. Eng. Chem. 59 (2018) 425-439.

[23] L. Laffont, T. Hezard, P. Gros, L.E. Heimburger, J.E. Sonke, P. Behra, D. Evrard, Mercury(II) trace detection by a gold nanoparticle-modified glassy carbon electrode using square-wave anodic stripping voltammetry including a chloride desorption step, Talanta 141 (2015) 26-32.

[24] M.B. Gholivand, B. Geravandi, M.H. Parvin, Anodic stripping voltammetric determination of iron(II) at a carbon paste electrode modified with dithiodianiline (DTDA) and gold nanoparticles (GNP), Electroanalysis 23 (2011) 1345-1351.

[25] F. Li, D. Pan, M. Lin, H. Han, X. Hu, Q. Kang, Electrochemical determination of iron in coastal waters based on ionic liquid-reduced graphene oxide supported gold nanodendrites, Electrochim. Acta 176 (2015) 548-554.

[26] Y. Zhu, D. Pan, X. Hu, H. Han, M. Lin, C. Wang, An electrochemical sensor based on reduced graphene oxide/gold nanoparticles modified electrode for determination of iron in coastal waters, Sens. Actuators B Chem. 243 (2017) 1-7.

[27] H. Han, Y. Li, D. Pan, C. Wang, F. Pan, X. Ding, A novel stainless steel needle electrode based on porous gold nanomaterials for the determination of copper in seawater, Anal. Methods 11 (2019) 1976-1983.

[28] N. German, A. Ramanaviciene, A. Ramanavicius, Formation of polyaniline and polypyrrole nanocomposites with embedded glucose oxidase and gold nanoparticles, Polymers 11 (2019) 377.

[29] N. German, A. Popov, A. Ramanaviciene, A. Ramanavicius, Enzymatic formation of polyaniline, polypyrrole, and polythiophene nanoparticles with embedded glucose oxidase, Nanomaterials 9 (2019) 806.

[30] Y.T. Li, L.N. Tang, Y. Ning, Q. Shu, F.X. Liang, H. Wang, G.J. Zhang, In vivo monitoring of serotonin by nanomaterial functionalized acupuncture needle, Sci. Rep. 6 (2016) 28018.

[31] W. Wang, G. Xu, X.T. Cui, G. Sheng, X. Luo, Enhanced catalytic and dopamine sensing properties of electrochemically reduced conducting polymer nanocomposite doped with pure graphene oxide, Biosens. Bioelectron. 58 (2014) $153-156$.

[32] A. Bello, M. Giannetto, G. Mori, R. Seeber, F. Terzi, C. Zanardi, Optimization of the DPV potential waveform for determination of ascorbic acid on PEDOT-modified electrodes, Sens. Actuators B Chem. 121 (2007) 430-435.

[33] K.M. Manesh, P. Santhosh, A. Gopalan, K.P. Lee, Electrocatalytic oxidation of NADH at gold nanoparticles loaded poly(3,4-ethylenedioxythiophene)-poly (styrene sulfonic acid) film modified electrode and integration of alcohol dehydrogenase for alcohol sensing, Talanta 75 (2008) 1307-1314.

[34] S. Khan, A.K. Narula, Electrochemical and optical bimodal sensing of caffeic acid based on electrodes made from nanorods of AuNPs:PEDOT:PSS and bio-hybrid chitosan:PEDOT:PSS, New J. Chem. 41 (2017) 8927-8939.

[35] L.A. Mercante, M.H.M. Facure, R.C. Sanfelice, F.L. Migliorini, L.H.C. Mattoso, D. S. Correa, One-pot preparation of PEDOT:PSS-reduced graphene decorated with Au nanoparticles for enzymatic electrochemical sensing of $\mathrm{H}_{2} \mathrm{O}_{2}$, Appl. Surf. Sci. 407 (2017) 162-170.

[36] Y. Li, C.H. Hsieh, C.W. Lai, Y.F. Chang, H.Y. Chan, C.F. Tsai, J.A. Ho, L.C. Wu, Tyramine detection using PEDOT:PSS/AuNPs/1-methyl-4-mercaptopyridine modified screen-printed carbon electrode with molecularly imprinted polymer solid phase extraction, Biosens. Bioelectron. 87 (2017) 142-149.

[37] H. Han, D. Pan, Y. Li, J. Wang, C. Wang, Stripping voltammetric determination of lead in coastal waters with a functional micro-needle electrode, Front. Mar. Sci. 7 (2020) 196.

[38] X. Pang, C. Cui, M. Su, Y. Wang, Q. Wei, W. Tan, Construction of self-powered cytosensing device based on $\mathrm{ZnO}$ nanodisks@g- $\mathrm{C}_{3} \mathrm{~N}_{4}$ quantum dots and application in the detection of CCRF-CEM cells, Nano Energy 46 (2018) 101-109. 
[39] A. Ramanavicius, P. Genys, A. Ramanaviciene, Electrochemical impedance spectroscopy based evaluation of 1,10-phenanthroline-5,6-dione and glucose oxidase modified graphite electrode, Electrochim. Acta 146 (2014) 659-665.

[40] A. Ramanavicius, A. Finkelsteinas, H. Cesiulis, A. Ramanaviciene, Electrochemical impedance spectroscopy of polypyrrole based electrochemical immunosensor, Bioelectrochemistry 79 (2010) 11-16.

[41] M. Lin, H. Han, D. Pan, H. Zhang, Z. Su, Voltammetric determination of total dissolved iron in coastal waters using a glassy carbon electrode modified with reduced graphene oxide, Methylene Blue and gold nanoparticles, Microchim. Acta 182 (2014) 805-813.

[42] M.H. Pournaghi-Azar, B.M. Fatemi, Simultaneous determination of ferric, ferrous and total iron by extraction differential pulse polarography: application to the speciation of iron in rocks, Microchem. J. 65 (2000) 199-207.

[43] P.C. Nascimento, L.D. Del-Fabro, D. Bohrer, L.M. de Carvalho, M.B. Rosa, S. M. Noremberg, $\mathrm{Al}(\mathrm{III})$ and $\mathrm{Fe}(\mathrm{III})$ balance in hemodialysis treatment assessed via fluid analysis by adsorptive stripping voltammetry and UV sample digestion, Electroanalysis 20 (2008) 1078-1084.

[44] J. Santos-Echeandia, Direct simultaneous determination of Co, Cu, Fe, Ni and V in pore waters by means of adsorptive cathodic stripping voltammetry with mixed ligands, Talanta 85 (2011) 506-512.

[45] G. Lu, X. Yao, X. Wu, T. Zhan, Determination of the total iron by chitosan-modified glassy carbon electrode, Microchem. J. 69 (2001) 81-87.

[46] M. Lin, D. Pan, X. Hu, H. Han, F. Li, Titanium carbide nanoparticles/ion-exchange polymer-based sensorfor catalytic stripping determination of trace iron in coastal waters, Sens. Actuators B Chem. 219 (2015) 164-170.

[47] D.I. Anguiano, M.G. García, C. Ruíz, J. Torres, I. Alonso-Lemus, L. AlvarezContreras, Y. Verde-Gómez, E. Bustos, Electrochemical detection of iron in a lixiviant solution of polluted soil using a modified glassy carbon electrode, Int. J. Electrochem. 2012 (2012), 739408.
[48] E.M. Ghoneim, Simultaneous determination of Mn(II), Cu(II) and Fe(III) as 2-(5'bromo-2'-pyridylazo)-5-diethylaminophenol complexes by adsorptive cathodic stripping voltammetry at a carbon paste electrode, Talanta 82 (2010) 646-652.

Haitao Han is currently an assistant professor at Yantai Institute of Coastal Zone Research, Chinese Academy of Sciences. He earned his Ph.D. degree in 2020 at Yantai Institute of Coastal Zone Research, Chinese Academy of Sciences. His research interests include novel electrochemical sensors and trace metals analysis in coastal waters.

Dawei Pan is currently a professor at Yantai Institute of Coastal Zone Research, Chinese Academy of Sciences. He received the Ph.D. degree in 2007 at Hunan University. His research interests include the integration of electrochemical sensor system, electroanalysis of coastal environment's pollutants and nutrients, and interface electrochemistry.

Fei Pan earned his master's degree in 2017 at Yantai University. He is currently a Ph.D. student at Yantai Institute of Coastal Zone Research, Chinese Academy of Sciences. His scientific interests include electrochemical sensors and environmental analysis.

Xueping Hu is currently an assistant professor at Linyi University. She earned the Ph.D. degree in 2018 at Yantai Institute of Coastal Zone Research, Chinese Academy of Sciences. Now she is engaged in fabrication of novel electrochemical sensors for metals determination in environmental samples.

Rilong Zhu is currently an associate professor at Hunan University. He earned his Ph.D. degree in 2009 at Hunan University. His research interests include fabrication of novel modified electrodes and heavy metals analysis. 